\title{
Correction to: Rheological studies and optimization of Herschel-Bulkley flow parameters of viscous karaya polymer suspensions using GA and PSO algorithms
}

\author{
Geetanjali Chauhan $^{1} \cdot$ Amit Verma $^{1} \cdot$ Akashdeep Das $^{1} \cdot$ Keka Ojha $^{1}$
}

Published online: 24 January 2018

(C) Springer-Verlag GmbH Germany, part of Springer Nature 2018

\section{Correction to: Rheol Acta \\ https://doi.org/10.1007/s00397-017-1060-x}

The original version of this article unfortunately contained a mistake. Some major changes that were required in the figures and table were not implemented. The corrections were mentioned when the proofread was submitted. These changes were made to make the manuscript more readable and presentable in black and white print out for the readers.

Figure 2: Arrows in the flow chart are displaced.

Figure 3: The green line for $1 \mathrm{wt} \% \mathrm{KG}$ is not visible in $\mathrm{b} / \mathrm{w}$ print out and it has been changed to olive green colour.

Figure 4: The captions on the $\mathrm{X}$ and $\mathrm{Y}$ axis does not match a common style.
Figure 5: The green line for $1 \mathrm{wt} . \% \mathrm{KG}$ is not visible in $\mathrm{B} / \mathrm{W}$ printout and it has been changed to olive green colour.

Figure 7: A new Fig. 7b is given below.

Figure 8: The green line for $1 \mathrm{wt} \% \mathrm{Kg}$ is not visible in $\mathrm{B} / \mathrm{W}$ printout and it has been changed to olive green colour.

Table 5: SSE is the parameter for Power law not HerschelBulkley i.e. the current table shows 3 columns for Power law, which should be 4 column including SSE of the Herschel Bulkley.

The corrected Figs. 2-5, 7, 8 and Table 5 are given below. The original article has been corrected.

The online version of the original article can be found at https://doi.org/ 10.1007/s00397-017-1060-x

Keka Ojha

keka_ojha@yahoo.com

1 Department of Petroleum Engineering, Indian Institute of Technology (Indian School of Mines), Dhanbad, India 

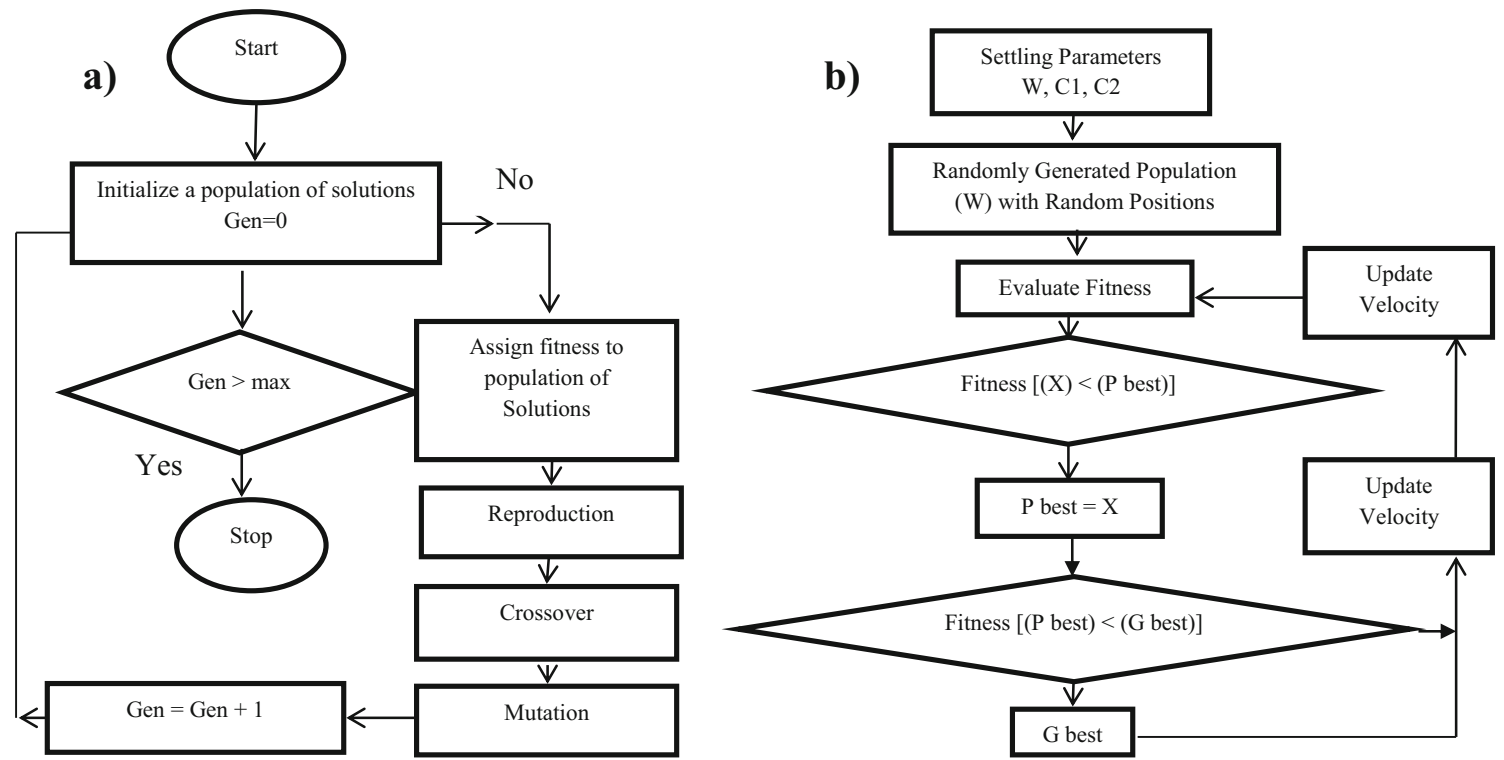

Fig. 2 Flowchart of genetic algorithm and particle swarm optimization algorithm (Rajendra and Pratihar 2011)

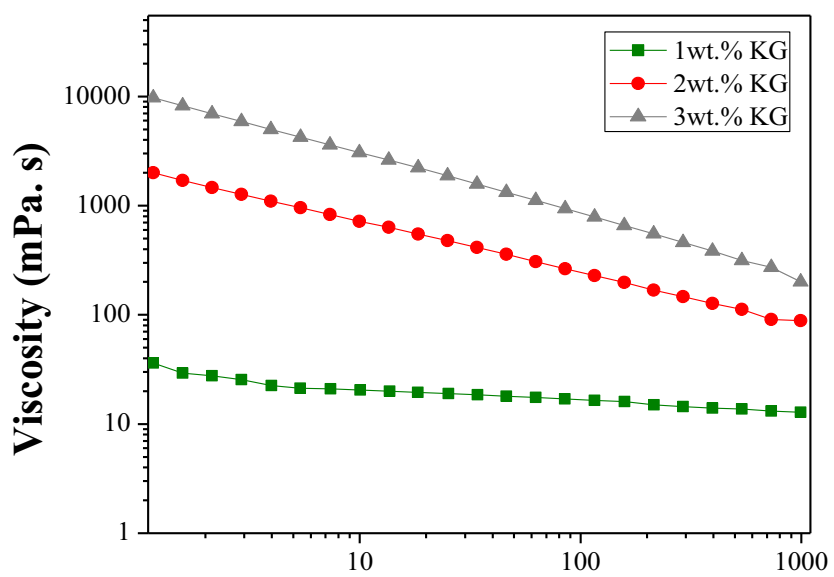

Shear Rate (1/s)

Fig. 3 Variation of viscosity with shear rate for 1,2 , and $3 \%$ karaya at $30{ }^{\circ} \mathrm{C}$ 

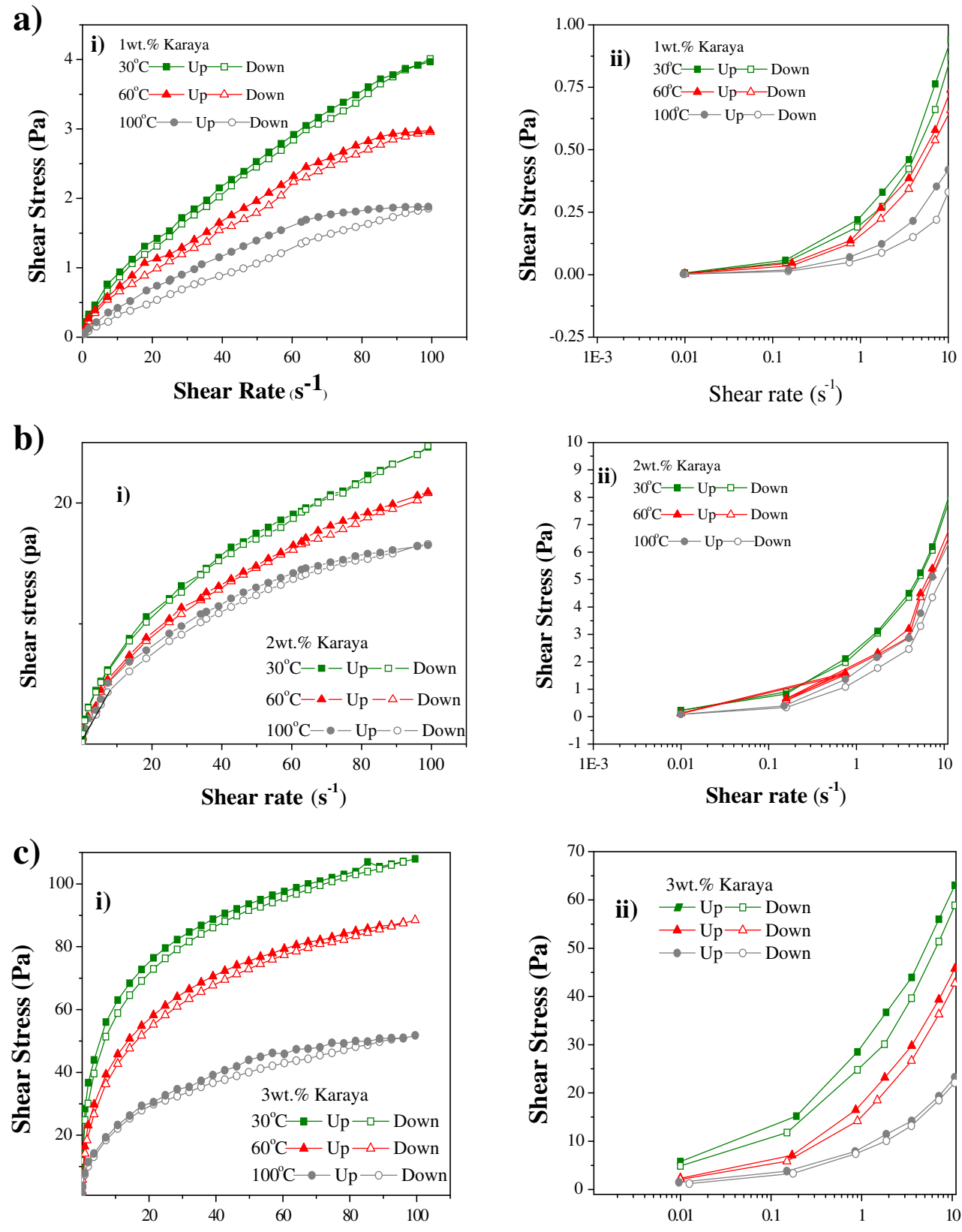

Shear Rate $\left(\mathbf{s}^{-1}\right)$

Fig. 4 a Hysteresis loop obtained for $1 \mathrm{wt} . \%$ Karaya at 30,60 and $100{ }^{\circ} \mathrm{C}$ i) at linear scale with rate varying from 0.01 to $100 \mathrm{~s}^{-1}$ ii) at logarithmic scale with shear rate varying from 0.01 to $10 \mathrm{~s}^{-1}$. b Hysteresis loop obtained for 2 wt.\% Karaya at 30,60 , and $100{ }^{\circ} \mathrm{C}$ i) at linear scale with

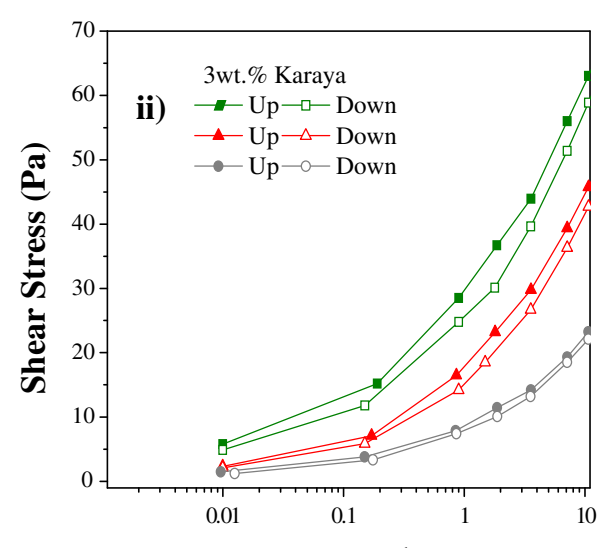

Shear Rate $\left(\mathbf{s}^{-\mathbf{1}}\right)$

shear rate varying from 0.01 to $100 \mathrm{~s}^{-1}$ ii) at logarithmic scale with shear rate varying from 0.01 to $10 \mathrm{~s}^{-1}$. c Hysteresis loop obtained for $3 \mathrm{wt} . \%$ Karaya at 30,60 , and $100{ }^{\circ} \mathrm{C}$ i) at linear scale with rate varying from 0.01 to $100 \mathrm{~s}^{-1}$ ii) at logarithmic scale with shear rate varying from 0.01 to $10 \mathrm{~s}^{-1}$ 


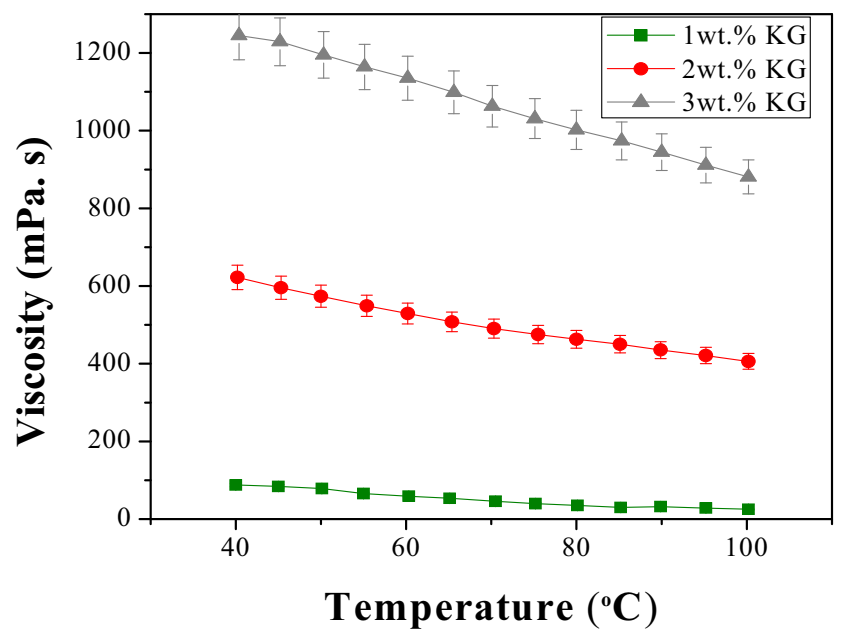

Fig. 5 Effect of temperature on viscosity at $100 \mathrm{~s}^{-1}$ shear rate
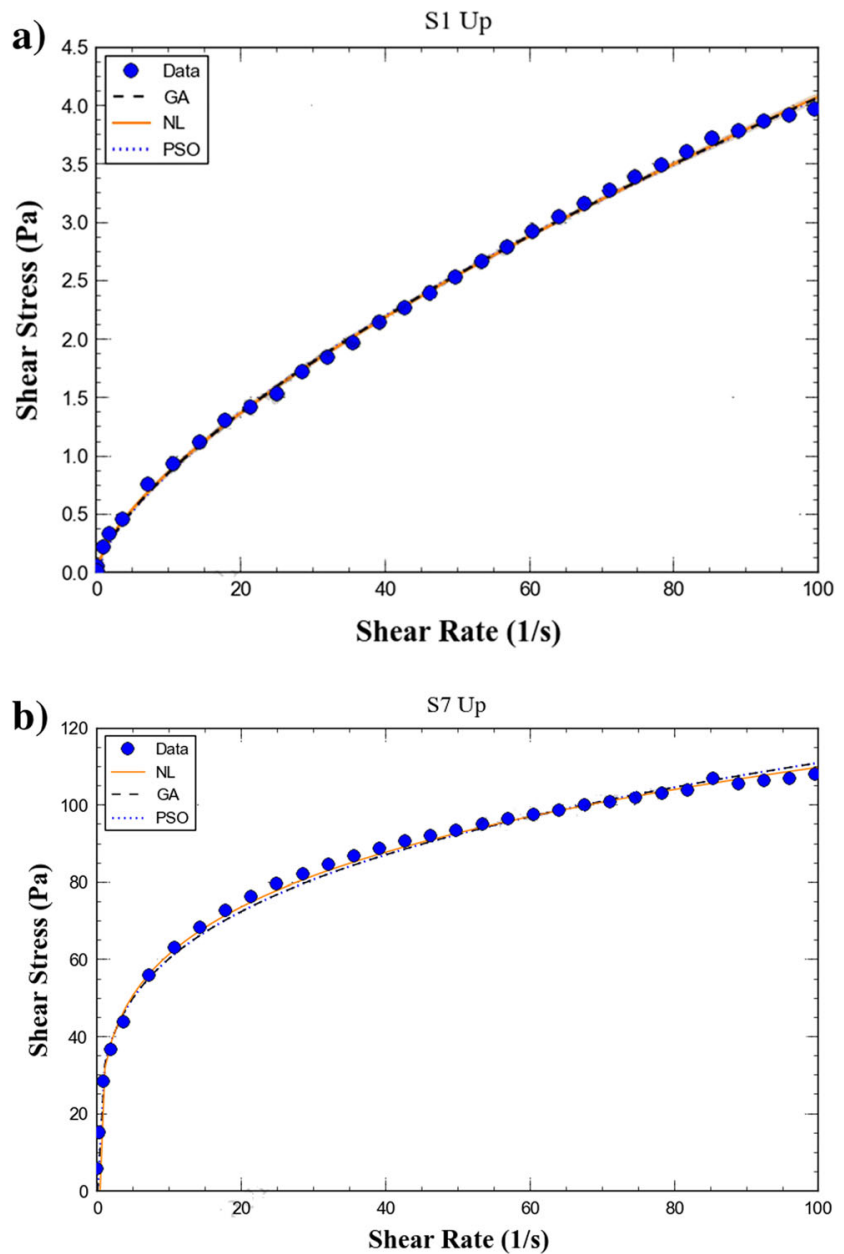

Fig. 7 a Superposition of rheological data and model curves obtained from using NL, GA and PSO method for sample S1-up. b Superposition of rheological data and model curves obtained from using NL, GA and PSO Method for sample S7-up

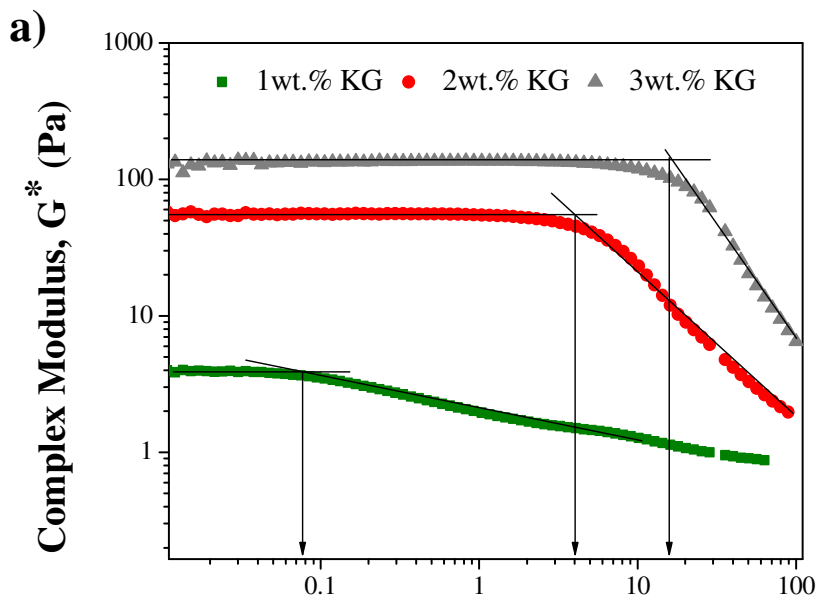

Shear Stress, Pa
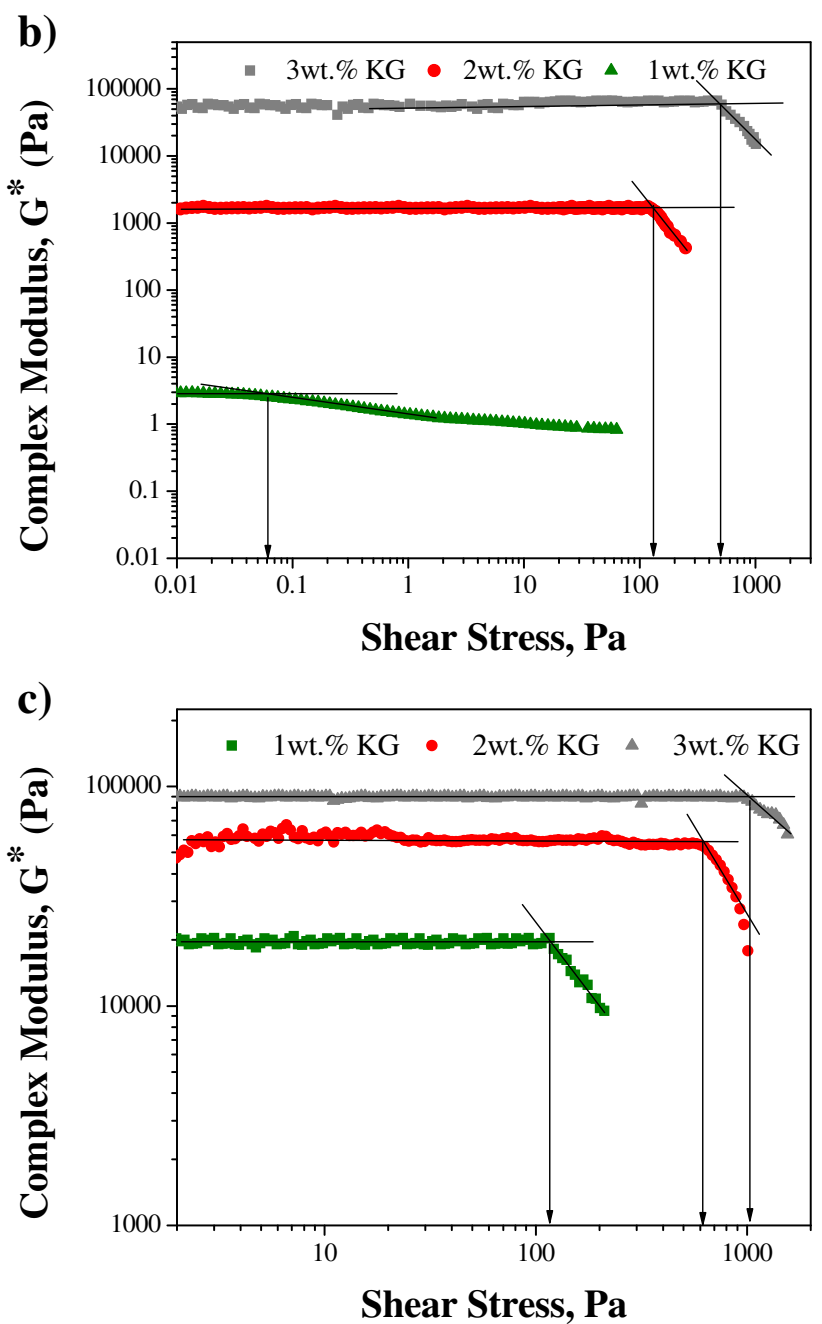

Fig. 8 a Estimation of yield stress using oscillatory study at $30^{\circ} \mathrm{C}$ for 1,2 and 3 wt.\% karaya suspensions at $1 \mathrm{~Hz}$ angular frequency. b Estimation of yield stress using oscillatory study at $60^{\circ} \mathrm{C}$ for 1,2 and $3 \mathrm{wt} \%$ karaya suspensions at $1 \mathrm{~Hz}$ angular frequency. $\mathbf{c}$ Estimation of yield stress using oscillatory study at $100^{\circ} \mathrm{C}$ for 1,2 and $3 \mathrm{wt}$. $\%$ karaya suspensions at $1 \mathrm{~Hz}$ angular frequency 
Table 5 The rheological parameters obtained for Power law and Herschel-Bulkley at different concentrations of gum karaya and temperatures with calculated coefficient of determination $\left(\mathrm{R}^{2}\right)$ and Sum of Square Error (SSE)

\begin{tabular}{|c|c|c|c|c|c|c|c|c|c|}
\hline \multirow[b]{2}{*}{ Sample } & \multicolumn{4}{|l|}{ Power law } & \multicolumn{5}{|c|}{ Herschel-Bulkley } \\
\hline & $\mathrm{K}_{(P L)},\left(\mathrm{Pa}-\mathrm{s}^{\mathrm{n}}\right)$ & $\mathrm{n}_{(P L)}$ & $\mathrm{R}^{2}$ & $\operatorname{SSE}\left(\mathrm{Pa}^{2}\right)$ & $\tau_{o(H B)}(\mathrm{Pa})$ & $K_{(H B)}\left(\mathrm{Pa}-\mathrm{s}^{\mathrm{n}}\right)$ & $n_{(H B)}$ & $R^{2}$ & $\operatorname{SSE}\left(\mathrm{Pa}^{2}\right)$ \\
\hline $1 \% 30^{\circ} \mathrm{C}$ up & 0.1847 & 0.6717 & 0.9989 & 0.0526 & 0.03996 & 0.171503 & 0.68621 & 0.9993 & 0.04779 \\
\hline $1 \% 30{ }^{\circ} \mathrm{C}$ down & 0.1509 & 0.7139 & 0.9993 & 0.034 & 0.046086 & 0.13757 & 0.73212 & 0.9994 & 0.0273 \\
\hline $1 \% 60{ }^{\circ} \mathrm{C}$ up & 0.1512 & 0.6594 & 0.9957 & 0.1266 & 0.017413 & 0.14521 & 0.66727 & 0.9957 & 0.1257 \\
\hline $1 \% 60{ }^{\circ} \mathrm{C}$ down & 0.1097 & 0.7241 & 0.9965 & 0.1015 & 0.043711 & 0.097535 & 0.74719 & 0.9966 & 0.0951 \\
\hline $1 \% 100{ }^{\circ} \mathrm{C}$ up & 0.1123 & 0.6333 & 0.9875 & 0.1691 & -0.06481 & 0.13685 & 0.5959 & 0.9883 & 0.1570 \\
\hline $1 \% 100{ }^{\circ} \mathrm{C}$ down & 0.0462 & 0.8085 & 0.9985 & 0.0166 & 0.005123 & 0.0451 & 0.81328 & 0.9985 & 0.0164 \\
\hline $2 \% 30^{\circ} \mathrm{C}$ up & 2.3507 & 0.5104 & 0.9995 & 0.802 & -0.1409 & 2.42946 & 0.5043 & 0.999 & 0.762 \\
\hline $2 \% 30{ }^{\circ} \mathrm{C}$ down & 2.186 & 0.525 & 0.9996 & 0.6215 & 0.03785 & 2.16601 & 0.52686 & 0.9996 & 0.618 \\
\hline $2 \% 60{ }^{\circ} \mathrm{C}$ up & 1.9026 & 0.5254 & 0.999 & 1.2587 & -0.2828 & 2.05508 & 0.510988 & 0.9991 & 1.0922 \\
\hline $2 \% 60{ }^{\circ} \mathrm{C}$ down & 1.804 & 0.5326 & 0.9989 & 1.2447 & -0.29368 & 1.95915 & 0.51717 & 0.9991 & 1.0610 \\
\hline $2 \% 100{ }^{\circ} \mathrm{C}$ up & 2.013 & 0.4697 & 0.9938 & 5.27 & -0.87257 & 2.5648 & 0.42616 & 0.9952 & 3.986 \\
\hline $2 \% 100{ }^{\circ} \mathrm{C}$ down & 1.609 & 0.516 & 0.996 & 3.3567 & -0.64211 & 1.96846 & 0.4788 & 0.9983 & 2.528 \\
\hline $3 \% 30^{\circ} \mathrm{C}$ up & 33.002 & 0.2637 & 0.9933 & 161.59 & -16.0517 & 47.98 & 0.2097 & 0.9972 & 67.704 \\
\hline $3 \% 30{ }^{\circ} \mathrm{C}$ down & 28.942 & 0.2913 & 0.9946 & 133.98 & -11.3434 & 39.1828 & 0.24479 & 0.9973 & 66.982 \\
\hline $3 \% 60{ }^{\circ} \mathrm{C}$ up & 21.452 & 0.3165 & 0.9926 & 136.74 & -10.4852 & 30.5776 & 0.26072 & 0.9963 & 67.634 \\
\hline $3 \% 60{ }^{\circ} \mathrm{C}$ down & 18.930 & 0.3404 & 0.9946 & 105.37 & -7.98803 & 25.5414 & 0.29189 & 0.9972 & 53.442 \\
\hline $3 \% 100{ }^{\circ} \mathrm{C}$ up & 9.9636 & 0.3676 & 0.9934 & 47.546 & -2.6685 & 12.022 & 0.3362 & 0.9944 & 40.088 \\
\hline $3 \% 100{ }^{\circ} \mathrm{C}$ down & 8.846 & 0.3852 & 0.9984 & 10.362 & -1.9658 & 10.3305 & 0.3585 & 0.9990 & 6.235 \\
\hline
\end{tabular}

